\title{
Promise of Mesenchymal Stem Cell Lysates in IBD Therapy: Are the Parts Greater than the Whole?
}

\author{
Victoria Gudiño ${ }^{1} \cdot$ Azucena Salas $^{1}$
}

Published online: 20 July 2020

(c) Springer Science+Business Media, LLC, part of Springer Nature 2020

Inflammatory bowel diseases (IBD) are chronic inflammatory disorders that include ulcerative colitis (UC) and Crohn's disease (CD). Both are relapsing diseases with similar symptomatology, although there are differences in terms of disease location and the type of mucosal inflammation: $\mathrm{UC}$ is characterized by superficial erosions and ulceration of the colonic mucosa, whereas CD can affect deeper layers of the entire intestinal wall and, in some patients, can induce development of fistulas or intestinal fibrosis. Their etiology is unknown, with current treatments that include anti-inflammatory or immunosuppressive drugs such as corticosteroids, antimetabolites such as azathioprine or methotrexate, biologics such as anti-tumor necrosis factor alpha (TNF $\alpha)$ or anti- $\alpha 4 \beta 7$ integrin antibodies, and more recently tofacitinib (a small molecule Janus kinase inhibitor). Despite the use of available therapeutic approaches and even surgery to treat complications such as stricturing (narrowing of the intestinal lumen) or penetrating (fistulizing) disease, a significant percentage of patients relapse or remain active. Furthermore, long periods of treatment exposure to immunosuppressant drugs can increase the risk of infections, cardiovascular diseases, or even cancer [1]. Hence, the need for alternative therapies that can help manage refractory patients remains an unmet need. In recent years, a potential therapeutic option without apparent systemic immunosuppressive side effects has emerged: mesenchymal stem cells (MSCs).

MSCs are multipotent progenitor cells capable of differentiating into limited cell lineages. In the last decades, they have garnered attention in the regenerative medicine field

Azucena Salas

ASALAS1@clinic.cat

Victoria Gudiño

GUDINO@clinic.cat

1 Inflammatory Bowel Disease Unit, Department of Gastroenterology, Instituto de Investigaciones Biomédicas August Pi i Sunyer, CIBER-EHD, Rosselló 149-153, 08036 Barcelona, Spain as they can be easily obtained from different tissues and expanded in vitro. More importantly, MSCs are considered immune-privileged since they express low levels of HLA class I molecules, as well as no HLA class II or co-stimulatory molecules (cluster of differentiation [CD]80, CD86, or CD40) under normal circumstances, enabling the use of allogeneic MSCs without rejection or prior immune ablation. In addition to their regenerative capacity, they possess intrinsic anti-inflammatory and immunomodulatory properties, which have been largely explored in inflammatory disorders such as IBD [2]. Compared to immunosuppressive treatments that have systemic effects, MSCs can presumably act locally at injury sites, potentially lowering the adverse effects associated with their use. To date, the use of MSC-based therapy is approved for treating perianal fistulizing $\mathrm{CD}$. While other therapeutic attempts have failed to promote complex fistula closure, expanded adipose-derived MSCs (Cx601, TiGenix) achieved remission in 50\% of patients at week 24 (compared with $34 \%$ in the placebo group) in a large-scale phase III clinical trial. Importantly, this effect was sustained in the vast majority of patients after 1 year of Cx601 injections [3]. In contrast, clinical trials addressing the use of MSCs for luminal UC or CD have not yielded conclusive results, and most have been discontinued at phase II/III [4]. Hence, although administration of MSCs is safe, evidence supporting their efficacy in placebo-controlled trials remains lacking. Likewise, their biological function and mechanisms of action are not completely understood, which further limits their suitability for clinical translation.

Two complementary models have been suggested to test the potential immunomodulatory effect of MSCs. On the one hand, MSCs could home to inflamed areas and enhance the stem cell regenerative capacity of intestinal cells onsite, while suppressing local inflammation [5].On the other hand, MSCs may release immunomodulatory factors that mediate their immunosuppressive function. Of the two models, increasing evidence supports the contribution of MSCderived mediators, especially since MSCs have an overall 
low engraftment capacity. Interestingly, the clearance of dead MSCs by phagocytic cells promotes a macrophage switch from an inflammatory (M1) to an anti-inflammatory (M2) phenotype [6]. MSCs can also promote M2 conversion independently of MSC engraftment and efferocytosis through the release of soluble factors such as prostaglandin E2 (PGE2) or TNF-induced protein 6 (TSG-6) [7], and they can influence other immune cell populations through the secretion of IL-10 or TGF $\beta$.

Studies in IBD mouse models have been essential to characterize the mechanisms of action of MSC. In the dextran sodium sulfate (DSS)-induced colitis mouse, injection of bone marrow-derived MSCs (BM-MSCs) improved colitis through their ability to secrete TSG-6, an anti-inflammatory factor capable of regulating matrix organization/function [7]. Interestingly, although some BM-MSCs are localized in the colon, most injected cells form lymphoid-like aggregates within the peritoneal cavity containing MSCs and immune cells, which reduce intestinal inflammation by suppressing $\mathrm{T}$ cell proliferation and inducting Tregs.

Thus, given the evidence supporting the contributions of MSC-derived factors, and the poor engraftment of MSC in tissues, an alternative approach might consist of studying factors produced by these cells. In that context, Liu et al. tested the efficiency of BM-MSCs-derived exosomes (MSC-Exos) on several models of induced-murine colitis. Exosomes are small lipid vesicles secreted by cells that are thought to facilitate intercellular communication. Mice administered MSC-Exos showed improved disease scores and lower secretion of pro-inflammatory cytokines [8]. Interestingly, those exosomes mostly targeted colonic macrophages of the inflamed mucosa, driving their conversion to an M2 phenotype that consequently produced high amounts of the anti-inflammatory cytokine IL-10. These findings support the potential efficacy of cell-free MSC approaches to promote mucosal immune modulation.

Following this strategy, the work by Nishikawa et al. [9] published in this issue of Digestive Diseases and Sciences describes the effect of filtered adipose tissue-derived MSC lysate (FADSTL) on a DSS-driven mouse model of colitis. They observed that repeated administration of FADSTL reduced signs of inflammation, significantly reducing body weight loss compared to the control group (75\% vs. $46.7 \%$, respectively). Consistently, FADSTL-treated mice showed a preserved intestinal epithelial architecture and reduced infiltration of neutrophils compared with controls. Both epithelial crypt destruction and accumulation of polymorphonuclear cells are distinctive characteristics of acute colitis that closely correlate with disease severity. As such, FADSTLtreated mice experienced a lower mortality rate and reduced expression of pro-inflammatory cytokines, while maintaining expression of tight junction structural proteins such as claudin-2 and occludin. Overall, their results indicate that repeated FADSTL administration prevented the development of acute colitis, thus providing a protective effect.

Previous studies have reported the regenerative and antiinflammatory effects of MSC lysates in other disease mouse models, such as those for acute liver failure and ischemia [10]. The study by Nishikawa et al. is the first to assess the efficiency of MSC lysates on a model of intestinal inflammation. Unlike cell-free MSC-conditioned medium and MSCExos, MSC lysates potentially contain MSC cell-surface proteins, which might amplify their beneficial effects. Nonetheless, this approach presented some drawbacks that need to be taken into consideration. FADSTL, as well as other cell-free therapies such as MSC-Exos, required continued administration, indicating a poor or nonexistent long-lasting effect that could complicate their feasibility in clinical settings. Moreover, the authors did not characterize either the factors contained in the lysate or its mechanism of action. Thus, despite the positive results, further detailed analyses are required before contemplating FADSTL as a potential therapeutic option for IBD. This type of analysis would be required not only for elucidating its mechanism of action, but also for ensuring homogeneity in FADSTL-produced batches.

In summary, although MSCs have been administered in mice via systemic routes, or locally to promote tissue regeneration and modulate inflammation, their clinical efficacy has only been proven in the case of perianal $\mathrm{CD}$, where intrafistula administration is approved to treat refractory penetrating disease [3]. An alternative to whole-cell administration is the use of MSC-conditioned medium, exosomes or, as in the study by Nishikawa et al., whole MSC lysates. The main advantages are easy distribution and access to tissues (no cell trapping in the lung, for instance). The rationale behind the use of MSC-secreted or -soluble components stems from their presumed potential to exert anti-inflammatory, anti-apoptotic, and regenerative effects. Nonetheless, increased evidence describing the active components present in MSC-derived factors will be required in order to move these approaches to a clinical setting.

\section{References}

1. Beaugerie L, Kirchgesner J. Balancing benefit vs risk of immunosuppressive therapy for individual patients with inflammatory bowel diseases. Clin Gastroenterol Hepatol. 2019;17:370-379.

2. Dave M, Mehta K, Luther J, Baruah A, Dietz AB, William AF Jr. Mesenchymal stem cell therapy for inflammatory bowel disease. Inflamm Bowel Dis. 2015;21:2696-2707.

3. Panés J, García-Olmo D, Vann Assche G, et al. Long-term efficacy and safety of stem cell therapy (Cx601) for complex perianal fistulas in patients with Crohn's disease. Gastroenterology. 2018;154:e4. 
4. Galipeau J, Sensébé L. Mesenchymal stromal cells: Clinical challenges and therapeutic opportunities. Cell Stem Cell. 2018;22:824-833.

5. Jung KH, Song SU, Yi T, et al. Human bone marrow-derived clonal mesenchymal stem cells inhibit inflammation and reduce acute pancreatitis in rats. Gastroenterology. 2011;140:e4.

6. Carty F, Mahon BP, English K. The influence of macrophages on mesenchymal stromal cell therapy: Passive or aggressive agents? Clin Exp Immunol. 2017;188:1-11.

7. Sala E, Genua M, Petti L, et al. Mesenchymal stem cells reduce colitis in mice via release of TSG6, independently of their localization to the intestine. Gastroenterology. 2015;149:e20.

8. Liu H, Liang Z, Wang F, et al. Exosomes from mesenchymal stromal cells reduce murine colonic inflammation via a macrophagedependent mechanism. JCI Insight. 2020;4:e131273.
9. Nishikawa T, Maeda K, Nakamura M, et al. Filtrated adipose tissue-derived mesenchymal stem cell lysate ameliorates experimental acute colitis in mice. Dig Dis Sci. (Epub ahead of print). https ://doi.org/10.1007/s10620-020-06359-3.

10. Jiao J, Milwid JM, Yarmush ML, Parekkadan B. A mesenchymal stem cell potency assay. Methods Mol Biol. 2011;677:221-231.

Publisher's Note Springer Nature remains neutral with regard to jurisdictional claims in published maps and institutional affiliations. 